\title{
44619 - ANESTHETIC MANAGEMENT OF A PARTURIENT WITH MARFAN SYNDROME
}

\section{CASE REPORT or SERIES}

\author{
Sudha Singh, Dept of Anesthesia, St. Joseph's Hospital, London, ON, Canada; \\ Chris Brooks, Anesthesia, St Joseph's Hospital;
}

PURPOSE: Anesthetic management of parturients with Marfan syndrome can be challenging because of cardiovascular and skeletal abnormalities (1). Parturients with aortic root dilatation are at risk for dissection (2). We describe the anesthetic management of a parturient with Marfan syndrome and aortic root dilatation having Cesarean delivery.

CLINICAL FEATURES: Patient consent was obtained. A 33 yr-old G1P0 woman at 26 wks gestation with Marfan syndrome was referred to the Anesthesia Clinic. She was known to have aortic root dilatation of $41 \mathrm{~mm}$ and mild mitral valve prolapse/regurgitation. She also had partial correction of severe scoliosis with Harrington rod placement from T3-L5 and total hip arthroplasty due to joint instability. Medications included metoprolol $100 \mathrm{mg}$ BID. Her cardiologist advised serial echocardiograms during pregnancy and Cesarean delivery to decrease risk of aortic dissection. The patient preferred general anesthesia for delivery. We requested a multidisciplinary conference including cardiac surgery. It was decided to proceed with Cesarean delivery at 38 wks gestation at the local cardiac surgery centre. The patient remained stable during pregnancy and her serial echoes remained unchanged. On the morning of surgery she was treated with antibiotics, metoprolol, ranitidine, and metoclopramide and brought to the cardiac OR. The obstetrical, neonatal, and cardiac surgery teams were present. She was lightly sedated with remifentanil while the BIS monitor, radial arterial line, and central lines were placed in addition to regular monitors. After preoxygenation, she underwent a modified rapid sequence induction using remifentanil, propofol and succinylcholine. Anesthesia was maintained with remifentanil, N20, O2, sevoflurane, and rocuronium. Intraop.TEE confirmed aortic root dilatation and mild mitral regurgitation. The patient remained hemodynamically stable. After delivery of the baby, an oxytocin infusion and morphine were given. The baby's Apgar scores were 4 and 8 . The baby required assisted bag-mask ventilation for the first minute only. The patient was transferred to the ICU for 24 hours and then to the CCU for the reminder of her hospital stay which was uncomplicated. Postoperative pain was managed with PCA morphine and regular acetaminophen. An echocardiogram done prior to discharge was unchanged. Both mother and baby did well.

CONCLUSIONS: The hemodynamic changes of pregnancy and delivery can be life-threatening to the parturient with Marfan syndrome. The successful outcome in this case was largely possible due to the multidisciplinary team approach used and regular follow up of the patient during her pregnancy and post-delivery. Anesthetic goals for delivery included minimizing aortic root shear forces and wall stress through invasive monitoring, intraoperative TEE, pharmacological treatment, and pain management. 
REFERENCES: (1). Obstet \& Gynecol. Survey 2003; 58: 800-3. (2) Eur. Heart J. 2005; 26:91420. 